\title{
Making paediatric assessment in suspected sexual abuse a therapeutic experience
}

\author{
Camille De San Lazaro
}

Sexual abuse in children may come to light in a variety of clinical settings. This paper outlines an approach to paediatric assessment in situations where a child has made a statement alleging abuse. Such children may be brought to the attention of a physician by referral from a general practitioner or from a child protection agency. In these situations a therapeutic approach is usually not expected and is rarely demanded by other professionals. While a carefully structured approach to the examination is needed to meet the requirements of social workers and the police who may be under considerable pressure to resolve the immediate child protection issues, the assessment should at all times be a healing experience for both the child and the family. A calm, gentle, and unhurried approach will go a long way toward making the examination part of the recovery process rather than another form of assault. Understanding of the special needs of such children is essential and paediatricians must always be sensitive to issues such as power, trust, and control.

\section{How can the paediatric assessment be therapeutic?}

Parents and children often have preconceived ideas about 'the medical'. Mothers, especially those who have experienced unpleasant gynaecological or obstetric procedures, will have deep anxieties, coloured by images of stirrup examinations and instrumentation. Some social workers harbour similar reservations and may be unable to reassure the child. The anogenital examination may be perceived as the most important part of the paediatric assessment and seen as an inevitably detrimental procedure. The paediatrician must use a structured system of approach which will quickly defuse anxiety and which at completion will leave the family and child happier and stronger, and on the path to 'recovery'.

\footnotetext{
'The Lindisfarne Centre', Department of Child Health, University of

Newcastle upon Tyne, Newcastle upon Tyne NE1 4LP

Correspondence to: Dr De San Lazaro.
}

\section{Taking the history}

The starting point is taking the medical history, and the child should participate in this whenever possible. If done correctly, the family will receive the message that the paediatrician is interested and cares in a holistic way. Recall of events from birth and infancy can be a delightful new experience for a child. The developmental history will highlight areas of achievement. Discussion of the child's likes or dislikes and previous illnesses allows the child an opportunity to engage in non-intrusive conversation and helps towards a sense of self importance and self value. The history taking allows the child and family to unwind and offers an opportunity to view abusive events within a much larger perspective.

While taking the history the paediatrician must be sensitive to the needs of either child or parent for privacy. There may be times when the parents do not want to be open, for example when discussing the family tree. Prefacing questions by asking 'Do you think you are able to talk about this here or would you rather we waited?' offers both parties a choice. When the parent's attitude is hostile or derogatory towards the child, firm intervention is needed, and the paediatrician may propose engaging with the child and family separately.

The history of the abusive event may be taken from the child, parent, or child protection workers. In our practice, children are always asked if they wish to talk about this themselves and if they decline they are encouraged to name the adult who will give the history, and are invited to listen and participate if they wish. Care must be taken to avoid leading questions and in our experience it is relatively easy to direct the parent or other adult towards doing little more than 'kick starting' the story, getting the child to fill in gaps with free recall. Opportunity to clay model or paint in another part of the room is offered; children often find they can participate in history taking when they are a little distance away and when they can choose to interject or respond to clarifying questions from a less intimidating position. A paediatrician who tells a child that 'many children have come here to talk to me about scary or sad things that have happened to them' is saying to the child that they are not alone and that their story will be accepted. The paediatrician's non-committal manner and lack of surprise or horror is likely to prevent retreat on the part of the child.

Care must be taken to ensure that history taking does not inflict distress, that children can retreat wholly if they wish, and that graphic detail is not demanded. It is usually sufficient to acquire basic facts, particularly relating to timing and chronicity, and any symptoms immediately attributable to trauma. 
The assessment procedure

Assessment includes observation through interview and play, and comprehensive physical examination in most cases. Engagement in play creates an atmosphere of fun and welcome. It also allows for extensive operation of choice for the child and provides the paediatrician with an opportunity to observe themes of play, motor skills, and language development.

Physical examination is not always required and the decision to proceed should be based both on history and symptoms. It is important that the child, the family, and the child protection agencies are briefed about the limitations of such examinations. There are often no clear signs of trauma in children who have given clear accounts of painful intrusive abuse. Conversely, there may be surprisingly gross signs of trauma in a child giving a minimal account of abuse, suggesting that the child may have a lot more to tell.

The process of physical examination should build on themes of wholeness, empowerment, and self worth. Introducing the child to clinical paraphernalia, for example the auroscope, the ophthalmoscope, or the magnifying light, and allowing the child to experiment and choose implements is usually the best way to begin. Dozens of choices may be offered from 'where shall we begin? - where next?' to 'which stethoscope?', 'which ear first?', 'who sits with the child?', 'which pillow or cushion?', 'bed screens drawn or open?'.

Anogenital inspection comes as part of a natural expectation in the process of systems evaluation or the head-to-toe approach. Distracting the child during the process with conversation (anything from pop groups, TV programmes, trainer shoes, or hairstyles) will diffuse embarrassment and help relaxation. A comment from the child like 'is that all?' should provide the examiner with gratifying evidence of their sensitivity and skill. The participation of the child throughout the process is critical for good outcome and opportunities for such involvement should be built into the systematic approach.

The process of communicating with the child about the examination outcome is the most significant element of the healing process. A matter-of-fact manner during a brief running commentary, for example, 'this looks fine', 'have you any other bruises for me to measure?' together with some fun questions -

Areas of information covered by the sexual abuse document used in Newcastle upon Tyne

1. Information about the child including problems during pregnancy and birth, illnesses and other adverse life events, motor and speech development, toilet training and school performance

2. An understanding of the family, its systems, levels of family support, financial and socia situation, health of parents and siblings, and existing stresses

3. Eventon, health of parents and siblings, and existing stresses each of these and time of most recent episode. Details of witnesses and any threats

4. Situation at the time of consultation, current placement, legal orders in place, health of the child, recent stooling, micturition, and menstruation

5. Assessment through observation - for example, level of engagement, behaviour, eye contact, symptoms of anxiety, fear, themes of play

6. Thorough physical examination of all systems, including meticulous growth measurements, anogenital inspection, and pubertal staging

7. Investigations - including forensic sampling, photography, and screening for sexually transmitted disease - when indicated

8. A 'wrap up' session, discussion of the findings with the child and family, arrangements for after care and review
'I hope you haven't got smelly socks' - keeps the child interested and entertained. Information about the anogenital inspection is given in a matter-of-fact way, choosing language with care, for example, 'I can see a little scratch here but I have had to use the special light because you are getting better so fast'.

The child who is expecting a shocked reaction from the doctor and who has dreaded the discovery of some horrible physical distortion has her self esteem restored not just by the right comment but more importantly by the paediatrician's calm non-reaction. A child who believes she exists in the mind of an adult only as a sexual tool can be assisted towards a sense of wholeness and bodily integrity by an interested and thorough examination of all systems in which anogenital review is a fleeting stage sandwiched into far more lengthy and more absorbing systems evaluation. Participation in measuring and charting bruises and scratches, colouring-in body charts, and eliciting tendon reflexes are both enjoyable and fascinating aspects of the clinical examination.

The 'wrap up' session includes a formal discussion of the findings with the caregiver and child. The physician must chose language with care. Words like 'little cut', 'scratch', 'bump' as opposed to 'tears' and 'scarring' are well within the child's grasp and also provide the correct perspective. The hymen is a ridiculously small piece of tissue and virtually no functional implications arise from most examinations for sexual abuse. Older children may wish to discuss the findings in privacy and should be offered the opportunity to do this. This time should also be used to discuss with the child and family the likely content of the medical report, recommendations to child protection agencies, and arrangements for after care.

Many children feel a sense of anticlimax and disappointment if the wrap up session is too brief. A further round of refreshments, planning, and completing play tasks will help draw a session to a more natural closure.

\section{Documentation}

When a child makes an allegation of sexual abuse it is highly likely that the legal system will become involved. The medical records must be amenable to future scrutiny and information should be gathered meticulously and recorded without ambiguity. For one child an abusive incident may be a single unforeseen event. For another, sexual abuse may form part of a spectrum of abuse and disadvantage. In the latter case vulnerability may well be reflected in previous health records, for example poor growth, non-attendance for medical appointments, previous referrals to hospital for non-organic symptomatology or symptoms relating to the urogenital tract. It is important that such potential evidence is sought out and carefully collated.

The paediatrician is an advocate for the child and has an important duty to consider all available health information if a comprehen- 
sive formulation is to be made about future risks. Failure to take such an overview may well leave the doctor liable to criticism both in the civil and criminal judicial systems. The medical record needs to be structured in such a way that medical reports are readily constructed and, if required, answers can be quickly found to questions in a court of law when the paediatrician is presenting evidence.

The safest way to ensure that all the needs of the child and family are comprehensively met and that potential requirements of the judicial process are safeguarded is to create a standardised documentary format. In Newcastle upon Tyne this structured approach has been formulated into a child protection record which includes the sexual abuse data document. The document allows space for verbatim recording of the history and detailed free notes of the physical findings, and also provides a multichoice checklist to ensure comprehensive collection of data. The different facets covered by this structured approach are given in the table. The Newcastle document has now been tested in over 1000 cases of alleged sexual abuse. In practice it provides the paediatrician with a system of safeguards because of the in-built checklist systems, particularly as the paediatrician may not always be in the position to judge which pieces of information will prove critical. In our experience this document also provides a tool for training and study, offering the trainee an idea of the scope of inquiry required in such situations and providing terms of reference for discussion and debate.

The personal impact of the tragedy of sexual abuse in a child can be overwhelming, even for the most experienced doctor. A familiar document which is frequently used can be a valuable asset. It helps the practitioner focus on the relevant issues and collate information in an orderly manner, contributing to a feeling of control. The medical assessment of the traumatised child may well be the watershed event in the multidisciplinary process. A system which allows the medical profession to feel confident will facilitate a reassuring and comforting atmosphere for the child and family and will thus contribute to a highly therapeutic outcome.

A copy of the sexual abuse document used in Newcastle upon Tyne can be obtained by writing to the author enclosing an A4 sized stamped addressed envelope. 